\title{
Témoignage de Martine Villelongue
}

Entretien

Sophie Cassagnes-Brouquet, Christine Dousset-Seiden, Michelle Zancarini-Fournel et Martine Villelongue

\section{OpenEdition}

Journals

Édition électronique

URL : http://journals.openedition.org/clio/10846

DOI : 10.4000/clio.10846

ISSN : $1777-5299$

Éditeur

Belin

Édition imprimée

Date de publication : 31 décembre 2012

Pagination : 203-208

ISSN : 1252-7017

Référence électronique

Sophie Cassagnes-Brouquet, Christine Dousset-Seiden, Michelle Zancarini-Fournel et Martine Villelongue, «Témoignage de Martine Villelongue », Clio. Femmes, Genre, Histoire [En ligne], 36 | 2012, mis en ligne le 31 décembre 2014, consulté le 19 avril 2019. URL : http://journals.openedition.org/ clio/10846 ; DOI : 10.4000/clio.10846 


\title{
Témoignage de Martine Villelongue
}

\author{
Entretien réalisé par Sophie CASSAGNES-BROUQUET, \\ Christine DOUSSET-SEIDEN \& Michelle ZANCARINI-FOURNEL
}

Entretien réalisé à Lyon, le 10 mai 2012, avec Martine Villelongue, directrice de l'université de la Mode. Historienne de l'Art, Martine Villelongue a soutenu une thèse sur l'auvre de Lucien Bégule (1848-1935), maître-verrier bonnais, à l'université de Lyon 2 en 1983, publiée en 2005'. Elle a ensuite travaillé pendant une quinzaine d'années au musée des Tissus et des Arts Décoratifs de Lyon: montage d'expositions et création du service des publics de ce musée 2 . Après avoir enseigné comme maître de conférences à l'université Lyon 2 en Histoire de l'Art où elle a crée les enseignements de l'Histoire des Textiles et de l'Histoire de la Mode, elle participe depuis 2003 à l'université de la Mode où elle poursuit ses enseignements et en assure la direction depuis 2009, tout en menant des recherches sur l'bistoire du vitrail ainsi que sur l'histoire de la mode et sur ses liens avec l'bistoire de l'art.

La place impartie à ce témoignage dans ce numéro ne nous a pas permis de publier l'intégralité de l'entretien. Les coupures sont signalées par (...)

\section{CLIO HFS : Pourquoi une université de la Mode?}

M.V. : L'université de la Mode est née, il y a vingt-deux ans, de la volonté du président de l'Université Lyon-2, de créer une filière professionnalisante en lien avec le tissu économique de la région. Lyon est une ville de tradition industrielle textile ancienne. L'université de la Mode est donc ancrée dans un environnement industriel et économique avec lequel elle a réussi à maintenir un lien pérenne malgré les changements très importants qu'a connus le

1 Villelongue 2005.

2 Villelongue 1981. 
secteur. Elle reçoit le soutien d'un certain nombre d'acteurs socioéconomiques dont le Musée des tissus créé par la Chambre de commerce sous le Second Empire (...)

Les débouchés offerts aux étudiant-e-s sont des postes de cadres dans tous les secteurs de la mode, vêtements bien sûr, mais aussi accessoires, cosmétiques, bijoux, design, etc. Les emplois visés sont également très variés, chefs de produit, assistants-directeurs de collection, marketing, relations publiques dans les secteurs de la culture, de l'audiovisuel, journalistes de mode ou encore professionnels du costume. Nous n'assimilons pas la mode au luxe et nos étudiants trouvent des débouchés aussi bien dans des marques de luxe que dans des entreprises tournées vers la grande diffusion (...)

CLIO HFS :Quel est le recrutement de l'université de la Mode?

M.V.: L'université accueille des étudiants désireux d'acquérir une initiation à la recherche, mais aussi des professionnels souhaitant mener un travail d'étude et de recherche en lien avec leur champ d'activités. Elle compte cette année cent vingt étudiants venant d'horizons très divers. En effet, son recrutement se veut international puisqu'un tiers au moins des étudiants en master 2 sont d'origine étrangère. L'université de la Mode a conclu de nombreuses conventions avec des universités étrangères et accueille des étudiants venus de toute l'Europe, mais aussi du Brésil, Mexique, de Chine, Japon, Russie, d'Europe Centrale, d'Algérie et de Tunisie. Les projets collectifs menés par les étudiants sont beaucoup plus intéressants s'ils sont de culture et de formation différentes. En revanche, son recrutement est très majoritairement féminin. Il y avait quatre garçons cette année, mais il arrive que certaines promotions soient entièrement féminines.

CLIO HFS : Quelle est votre approche de l'enseignement de l'histoire du costume et de la mode?

M.V. : (...) L'idée est de montrer que la mode est un objet historique et en lien avec l'histoire dans tous les sens du terme: sociologique, économique, histoire de l'art, histoire du genre, etc. Ce qui m'intéresse, c'est de décortiquer un vêtement, une parure et de dire comment ils ont été réalisés, avec quels matériaux, pour quelle 
personne et par qui, quel en a été le coût. Un vêtement, c'est un produit économique, c'est un produit artisanal, c'est un produit artistique. Parfois, c'est un produit industriel, mais à qui est-il destiné ? Représente-t-il le costume porté par tout le monde, ou au contraire s'agit-il d'une excentricité ? Est-ce que la presse de mode en a parlé et d'ailleurs est-ce qu'une presse de mode existait à l'époque ? Comment le vêtement a-t-il été diffusé ? Toutes ces questions nous intéressent et sont bien sûr mises en rapport avec la mode actuelle (...)

\section{CLIO HFS : Intégrez-vous les problématiques de genre dans vos} enseignements et les recherches des étudiants?

M.V.: Bien sûr, car les thématiques de genre sont au cœur du sujet de la mode. Par exemple, nous avons fait intervenir auprès de nos étudiants Farid Chenoune, historien de la mode, professeur à l'École Nationale Supérieure des Arts Décoratifs et à l'Institut Français de la Mode, l'un des premiers historiens à s'intéresser à la mode masculine avec son livre Des modes et des hommes, deux siècles d'élégance masculine ${ }^{3}$ où il étudie la mode masculine de la fin du XVIII siècle à nos jours et met en avant la pression des codes vestimentaires de genre. Mais il s'intéresse aussi aux normes de la féminité dans Les dessous de la féminitét $^{4}$ : il est venu donner une conférence à nos étudiants intitulée "Les dessous de la féminité, un siècle de lingerie ».

Il faut souligner que parmi le petit nombre d'étudiants masculins inscrits à l'université de la Mode, certains se montrent très concernés par le sujet. Par exemple, un étudiant a réalisé un mémoire de recherche sur la création actuelle de mode masculine à Berlin. Les étudiant-e-s ont aussi organisé un café de la mode intitulé «Attention, les hommes nous piquent nos fringues!». Cet intérêt pour la mode masculine n'est pas un hasard. Depuis deux ans, elle bouleverse tous les codes de la mode. On pourrait penser qu'elle était, si j'ose dire négligée, en tous les cas moins présente dans les médias, mais ce n’est pas le cas; elle semble aujourd'hui placée au cœur de la mode et vient bouleverser certains stéréotypes de la masculinité.

3 Chenoune 1993.

4 Chenoune 1998. 
CLIO HFS : Prenez-vous également en compte les âges de la vie? M.V. : Assez peu dans les cours, si ce n'est pour dire qu'avec le début $\mathrm{du} \mathrm{XX}^{\mathrm{e}}$ siècle apparait une mode enfantine dans les élites, auparavant l'enfant est habillé comme les adultes, la mode enfantine en tant que telle est récente. Mais au niveau de la recherche, la mode enfantine actuelle intéresse quelques étudiants qui ont réalisé des mémoires sur certaines marques de mode spécialisées. Elle a aussi été abordée dans notre dernier colloque. Les travaux et les réalisations sur les seniors sont assez nombreux, comme ce défilé réalisé dans une maison de retraite. Plus fréquents sont les sujets de recherche sur la mode des jeunes et des adolescents, les groupes, les tribus, les groupes urbains, les lolitas, les skateurs, les surfeurs, etc.

CLIO HFS : Mode et vêtements entretiennent un rapport au corps évident, quelle est votre approche?

M.V. : Nous faisons intervenir au niveau du master 2 une spécialiste de Freud qui propose un cours sur l'analyse psychanalytique, elle parle beaucoup du genre et de la différence d'appréhension entre les hommes et les femmes. Une psychologue aborde plus précisément le rapport au corps dans son cours au travers d'une réflexion sur l'image de soi en tenant compte de la dimension historique. Je vous rappelle que les femmes n'ont des jambes que depuis 1916, auparavant elles les avaient toujours cachées. Si vous lisez les historiens du corps comme Georges Vigarello ${ }^{5}$, vous constatez que la mode médiévale et celle de la Renaissance privilégient le haut du corps, le décolleté, le haut du buste, le cou et le visage, transformant le corps de la femme en une sorte de pyramide servant de piédestal au visage, d'où le développement des cosmétiques à la Renaissance. L'image que l'on veut donner de son corps varie avec l'histoire, mais aussi avec la psychologie.

Cette dialectique entre le caché et le dévoilé, la mise en valeur de certaines parties du corps aux dépends d'autres, ce modelage du corps de la femme se maintient chez les créateurs de haute couture contemporains. On pourrait même les diviser en deux familles; la première c'est celle de Christian Dior, quelle que soit la femme et sa morphologie, elle doit s'adapter à un idéal féminin, petit buste, jupe

5 Vigarello 2005. 
évasée et deux petites jambes, un modèle poursuivi par Christian Lacroix en quelque sorte; tandis que Chanel privilégie l'élégance par le confort, une femme ne se sent élégante que si elle est à l'aise dans toutes ses activités. L'élégance passe par le confort, alors que pour Dior elle passe par la contrainte qui remodèle le corps comme au XVIII ${ }^{e}$ siècle, hanches élargies et buste triangulaire ${ }^{6}$.

CLIO HFS : Ces réflexions sur la mode, son histoire et son futur vous ont amené à organiser des colloques réguliers, pourtiezvous nous en dire plus?

M.V. : Tous les deux ans, depuis 2008, nous essayons d'organiser un colloque pour enrichir notre réflexion au-delà des rencontres et des tables rondes organisées avec des créateurs ou des professionnels de la Mode. Le premier en 2008 intitulé Voiles dévoilés, pudeur, foi et élégance, a, bien entendu, placé les questions de genre au cœur de nos réflexions, mais bien sûr elles ne se limitaient pas là; elles ont également porté sur le voile sous toutes ses formes, religieux, moral, politique ou historique 7 . En 2010, le colloque S'babiller pour travailler a aussi accordé une place importante à la question du genre. Sa volonté était d'établir une anthropologie du vêtement de travail en abordant ses divers aspects, sociologique, économique et esthétique, dans ses fonctions de symbole, d'image et de protection et ses valeurs dans l'histoire comme dans la mode contemporaine. Plusieurs contributions ont traité des vêtements de travail féminins, tantôt dans des milieux très féminisés: les hôtesses de l'air chez Sabena 19472001, l'infirmière hospitalière, etc., tantôt dans des milieux au contraire très masculins comme les femmes officiers de marine marchande qui doivent à certains moments adopter les codes masculins, tout en affichant leur identité de femmes ${ }^{8}$.

Le colloque organisé cette année au printemps par l'université, "Défier le temps une affaire de mode», portait sur les rapports entre la Mode et le Temps. Il était articulé selon trois axes : le passé et le futur, réservoirs de la créativité pour la mode; des temps de la vie au

\footnotetext{
Chenoune 2007.

Villelongue 2008.

8 Villelongue 2001.
} 
temps de la mode, le temps des garde-robes, les âges de la vie et de la mode, la temporalité de la mode; défier le temps, les réponses de la mode d'aujourd'hui. Bien que moins évident, le rapport masculin/féminin y a aussi été bien présent (...)

\section{Bibliographie}

Chenoune Farid, 1993, Des modes et des hommes, deux siècles d'élégance masculine, Paris, Flammarion.

—, 1998, Les dessous de la féminité, un siècle de lingerie, Paris, Assouline.

Vigarello Georges, Corbin Alain \& Jean-Jacques Courtine, 2005-2006, Histoire $d u$ Corps, Paris, Éd. du Seuil.

Villelongue Martine, 1981, Soierie lyonnaise, 1850-1940, Lyon, CNRS (avec Henriette POMmier \& Christian Roupioz).

—, 2005, Lucien Bégule, maître verrier lyonnais, Châtillon-sur-Chalaronne, Éditions de la Taillanderie.

—, (dir.) 2008, Les voiles dévoilés, pudeur, foi, élégance, Lyon, Éditions lyonnaises d'art et d'histoire.

—, (dir.) 2011, S'habiller pour travailler, colloque des 30 et 31 mars 2010, Lyon, Éditions lyonnaises d'art et d'histoire. 